\title{
Viable Insider Markets
}

\author{
Olfa Draouil ${ }^{1,2}$ and Bernt $\varnothing \mathrm{ksendal}{ }^{1,2}$
}

4 December 2018

MSC(2010): 60H10; 60H40; 60J65; 91B55; 91B70; 91G80; 93E20

Keywords: Stochastic differential equation; viable financial market; logarithmic utility; inside information; Donsker delta functional; Hida-Malliavin calculus; forward integrals; optimal insider control.

\begin{abstract}
In this paper we investigate a logarithmic utility maximization problem of the terminal wealth for an insider portfolio, where the inside information consists of knowledge of some future values of the Brownian motion $B(t)$ driving the financial market. More specifically, we assume that at time $t$ the insider has access to market information at least $\varepsilon_{t}>0$ time units ahead of time. We consider two cases:
\end{abstract}

- Case 1: $t+\varepsilon_{t}>T$.

In this case we assume that at time $t$ the insider knows the value of $B\left(t+\varepsilon_{t}+r\right) ; 0 \leq$ $r \leq \varepsilon_{0}-t-\varepsilon_{t}$, for some $\varepsilon_{0}>T$. More precisely, we assume that the inside information $\mathbb{H}$ is a progressive inside information flow:

$$
\mathbb{H}=\left\{\mathcal{H}_{t}\right\}_{t \in[0 . T]}, \quad \mathcal{H}_{t}=\mathcal{F}_{t} \vee \sigma\left(B\left(t+\varepsilon_{t}+r\right), 0 \leq r \leq \varepsilon_{0}-t-\varepsilon_{t}\right), \forall t \in[0, T],
$$

for $t+\varepsilon_{t}>T$, where $\mathcal{F}_{t}$ is the $\sigma$-algebra generated by $B(s) ; 0 \leq s \leq t$.

- Case 2: $t+\varepsilon_{t}<T$.

In this case we consider the enlarged filtration $\mathbb{H}=\left\{\mathcal{H}_{t}\right\}_{t \in[0 . T]}$ where $\mathcal{H}_{t}=\mathcal{F}_{t+\varepsilon_{t}}$.

The goal of this paper is to study when the market is viable, in the sense that the corresponding utility maximization problem admits a finite value.

To study this problem we first consider the case when the inside information at time $t$ is given by knowledge of the values of $Y_{t}=\left(B\left(t+\varepsilon_{t}^{(1)}\right), B\left(t+\varepsilon_{t}^{(2)}\right)\right)$ for $0<\varepsilon_{t}^{(1)}<\varepsilon_{t}^{(2)}, t \in$ $[0, T]$, and we use forward integrals, Hida-Malliavin derivatives and the Donsker delta

\footnotetext{
${ }^{1}$ Department of Mathematics, University of Oslo, P.O. Box 1053 Blindern, N-0316 Oslo, Norway. Emails: oksendal@math.uio.no, olfad@math.uio.no

${ }^{2}$ This research was carried out with support of the Norwegian Research Council, within the research project Challenges in Stochastic Control, Information and Applications (STOCONINF), project number $250768 / \mathrm{F} 20$.
} 
functional in our approach. In Case 1 we give a necessary condition for the viability of the market. We prove that the market is viable only if

$$
\int_{0}^{T} \frac{1}{\varepsilon_{t}} d t<\infty
$$

In Case 2 we give a sufficient and necessary conditions for the viablity and we prove that the market is not viable for any choice of $\varepsilon_{t}>0$.

\section{Introduction}

The problem of insider trading is studied in many works for example $[\mathrm{A}, \mathrm{AIS}, \mathrm{AI}, \mathrm{B} \emptyset, \mathrm{C}$, DMØP2, DØ1, H, IPW, PK]. This type of problems is related to the enlargement of filtration $[\mathrm{Ja}, \mathrm{J}, \mathrm{Je}, \mathrm{M}]$.

The purpose of this paper is to study the classical Merton problem of portfolio optimization in a financial market, extended to the case when the trader has inside information, i.e. has access to information about the value of random variables related to the terminal value $S(T)$ of the price of the risky asset.

We are using basically the same framework as in the paper $[\mathrm{PK}]$, but with a progressive inside information flow rather than a fixed initial inside information. In first time we consider the inside information given by

$$
\mathbb{H}=\left\{\mathcal{H}_{t}\right\}_{t \in[0 . T]}, \quad \mathcal{H}_{t}=\mathcal{F}_{t} \vee \sigma\left(B\left(t+\epsilon_{t}+r\right), 0 \leq r \leq \epsilon_{0}-t-\epsilon_{t}\right), \forall t \in[0, T],
$$

where $t+\epsilon_{t}>T$ and $t+\epsilon_{t}$ goes to $T$ when $t$ goes to $T$.

Here $B(t)$ is a Brownian motion on a given filtered probability space $\left(\Omega, \mathbb{F}=\left\{\mathcal{F}_{t}\right\}_{0 \leq t \leq T}, P\right)$, where $\mathcal{F}_{t}$ is the $\sigma$-algebra generated by $B(s) ; 0 \leq s \leq t$. Secondly we consider the enlarged filtration given by $\mathbb{H}=\left\{\mathcal{H}_{t}\right\}_{t \in[0 . T]}$ where $\mathcal{H}_{t}=\mathcal{F}_{t+\varepsilon_{t}}$ for $t+\varepsilon_{t}<T$.

For each $t \in[0, T]$ we fix a constant $\varepsilon_{t}>0$ and we let $Y_{t}$ be a given $\sigma\left(B\left(t+\epsilon_{t}\right)\right)$-measurable random variable representing the inside information available to the controller.

We consider the problem of optimal inside portfolio $\pi(t)=\pi\left(t, Y_{t}\right)$ in a financial market with a corresponding wealth process $X(t)=X^{\pi}(t)$ modelled by

$$
\begin{cases}d X(t) & =\pi\left(t, Y_{t}\right) X(t)[\alpha(t) d t+\beta(t) d B(t)] ; \quad t \in[0, T] \\ X(0) & =1 .\end{cases}
$$

where $\alpha$ and $\beta$ are $\mathbb{F}$-adapted bounded processes.

It is not clear if $B(t)$ is a semimartingale with respect to $\mathbb{H}$. Therefore we interpret the $d B$ - integral in equation (1.2) as a forward integral. See Section 2.

Let $\mathcal{A}$ be the set of $\mathbb{H}$-adapted, self-financing portfolios $\pi\left(t, Y_{t}\right)$ such that the forward equation (1.2) has a unique solution. We study the following insider optimal portfolio problem: 
Problem 1.1 Find $\pi^{*} \in \mathcal{A}$ such that

$$
J\left(\pi^{*}\right)=\sup _{\pi \in \mathcal{A}} J(\pi),
$$

where

$$
J(\pi):=\mathbb{E}\left[\log \left(X^{\pi}(T)\right)\right] .
$$

Definition 1.2 The market(1.2) is called viable if

$$
\sup _{\pi \in \mathcal{A}} J(\pi)<\infty .
$$

The purpose of this paper is to study under what inside information flow $\mathbb{H}$ the insider market is viable or not.

More precisely to study this problem we consider the inside information of the form $Y_{t}=\left(B\left(t+\varepsilon_{t}^{(1)}\right), B\left(t+\varepsilon_{t}^{(2)}\right)\right)$ for $\varepsilon_{t}^{(1)}<\varepsilon_{t}^{(2)}$. Using the Donsker delta functional of $Y_{t}$ and its Hida-Maliavin derivative we prove that the maximal expected logarithmic utility of the terminal wealth process depends only on the knowledge of $B\left(t+\varepsilon_{t}^{(1)}\right)$. Therefore we deduce that given the information $\sigma\left(B\left(t+\epsilon_{t}+r\right), 0 \leq r \leq \epsilon_{0}-t-\epsilon_{t}\right), \forall t \in[0, T]$ for the insider, the information of $B\left(t+\epsilon_{t}\right)$ is relevant.

When $t+\epsilon_{t}>T$ we give a necessary condition in terms of $\epsilon_{t}$ in order to obtain a viable market.

We show that the insider market is not viable if

$$
\int_{0}^{T} \frac{1}{\varepsilon_{t}} d t=\infty .
$$

This extends a result in $[\mathrm{PK}]$, where it is proved that if the insider knows the value of $B(T)$ already from time 0 (corresponding to the case $\varepsilon_{t}=T-t$ for all $t$ ), then the market is not viable.

Then we give some examples of characterization of viability.

Finally we treat the case where $t+\epsilon_{t}<T$ and $\mathcal{H}_{t}=\mathcal{F}_{t+\epsilon_{t}}$. We give a sufficient and necessary conditions in terms of $\epsilon_{t}$ for the viability of the market. We show that this insider market is viable if and only if $\int_{0}^{T} \frac{1}{\epsilon_{t}} d t<\infty$. Hence the market is never viable in this case.

This type of problem has been studied in the paper [DØPP] (see in particular Section 6.3), but the methods there are different and the results are no as explicit as in our paper. Also Corcuera et al. $[\mathrm{C}]$ treated this type of problem but using another kind of filtration called dynamical enlargement of filtration. This filtration consists in the knowledge of a functional of the underlying deformed by an independent noise process which tends to 0 as $T$ approaches. This type of information is different from the one that we consider in this paper. The technique also of treating the problem is different from $[\mathrm{C}]$.

This paper is organized as follows:

In Section 2, we give a brief review of forward integrals, Donsker delta functional and HidaMalliavin calculus. 
In Section 3 we consider the inside information $Y_{t}=\left(B\left(t+\varepsilon_{t}^{(1)}\right), B\left(t+\varepsilon_{t}^{(2)}\right)\right)$ for $\varepsilon_{t}^{(1)}<\varepsilon_{t}^{(2)}$ to give a necessary condition in terms of $\epsilon_{t}$ to study the viability of the market when $t+\epsilon_{t}>T$. Finally in Section 4 we consider the case where $t+\epsilon_{t}<T$ we give sufficient and necessary conditions for the viability of the market.

\section{Forward integrals and the Donsker delta functional}

\subsection{The forward integral with respect to Brownian motion}

Since we are not sure that $B(t)$ is a semimartingale under $\mathbb{H}$ or not we interpret the equation (1.2) as forward integrals. The forward integral with respect to Brownian motion was first defined in the seminal paper [RV] and further studied in [RV1], [RV2]. This integral was introduced in the modelling of insider trading in $[\mathrm{B} \varnothing]$ and then applied by several authors in questions related to insider trading and stochastic control with advanced information (see, e.g., [DMØP2]). The forward integral was later extended to Poisson random measure integrals in [DMØP1].

Definition 2.1 We say that a stochastic process $\phi=\phi(t), t \in[0, T]$, is forward integrable (in the weak sense) over the interval $[0, T]$ with respect to $B$ if there exists a process $I=$ $I(t), t \in[0, T]$, such that

$$
\sup _{t \in[0, T]}\left|\int_{0}^{t} \phi(s) \frac{B(s+\epsilon)-B(s)}{\epsilon} d s-I(t)\right| \rightarrow 0, \quad \epsilon \rightarrow 0^{+},
$$

in probability. In this case we write

$$
I(t):=\int_{0}^{t} \phi(s) d^{-} B(s), t \in[0, T]
$$

and call $I(t)$ the forward integral of $\phi$ with respect to $B$ on $[0, t]$.

The following results give a more intuitive interpretation of the forward integral as a limit of Riemann sums:

Lemma 2.2 Suppose $\phi$ is càglàd and forward integrable. Then

$$
\int_{0}^{T} \phi(s) d^{-} B(s)=\lim _{\triangle t \rightarrow 0} \sum_{j=1}^{J_{n}} \phi\left(t_{j-1}\right)\left(B\left(t_{j}\right)-B\left(t_{j-1}\right)\right),
$$

with convergence in probability. Here the limit is taken over the partitions $0=t_{0}<t_{1}<\ldots<t_{J_{n}}=T$ of $t \in[0, T]$ with $\triangle t:=\max _{j=1, \ldots, J_{n}}\left(t_{j}-t_{j-1}\right) \rightarrow 0, n \rightarrow \infty$.

Remark 2.3 From the previous lemma we can see that, if the integrand $\phi$ is $\mathcal{F}$-adapted, then the Riemann sums are also an approximation to the Itô integral of $\phi$ with respect to the Brownian motion. Hence in this case the forward integral and the Itô integral coincide. In this sense we can regard the forward integral as an extension of the Itô integral to a nonanticipating setting. 
We now give some useful properties of the forward integral. The following result is an immediate consequence of the definition.

Lemma 2.4 Suppose $\phi$ is a forward integrable stochastic process and $G$ a random variable. Then the product $G \phi$ is forward integrable stochastic process and

$$
\int_{0}^{T} G \phi(t) d^{-} B(t)=G \int_{0}^{T} \phi(t) d^{-} B(t) .
$$

The next result shows that the forward integral is an extension of the integral with respect to a semimartingale:

Lemma 2.5 Let $\mathbb{G}:=\left\{\mathcal{G}_{t}, t \in[0, T]\right\}(T>0)$ be a given filtration. Suppose that

1. $B$ is a semimartingale with respect to the filtration $\mathbb{G}$.

2. $\phi$ is $\mathbb{G}$-predictable and the integral

$$
\int_{0}^{T} \phi(t) d B(t)
$$

with respect to $B$, exists.

Then $\phi$ is forward integrable and

$$
\int_{0}^{T} \phi(t) d^{-} B(t)=\int_{0}^{T} \phi(t) d B(t)
$$

We now turn to the Itô formula for forward integrals. In this connection it is convenient to introduce a notation that is analogous to the classical notation for Itô processes.

Definition 2.6 A forward process (with respect to $B$ ) is a stochastic process of the form

$$
X(t)=x+\int_{0}^{t} u(s) d s+\int_{0}^{t} v(s) d^{-} B(s), \quad t \in[0, T]
$$

(x constant), where $\int_{0}^{T}|u(s)| d s<\infty, \mathbf{P}$-a.s. and $v$ is a forward integrable stochastic process. A shorthand notation for (2.7) is that

$$
d^{-} X(t)=u(t) d t+v(t) d^{-} B(t)
$$

Theorem 2.7 The one-dimensional Itô formula for forward integrals. Let

$$
d^{-} X(t)=u(t) d t+v(t) d^{-} B(t)
$$

be a forward process. Let $f \in \mathbf{C}^{1,2}([0, T] \times \mathbb{R})$ and define

$$
Y(t)=f(t, X(t)), \quad t \in[0, T] .
$$

Then $Y(t), t \in[0, T]$, is also a forward process and

$$
d^{-} Y(t)=\frac{\partial f}{\partial t}(t, X(t)) d t+\frac{\partial f}{\partial x}(t, X(t)) d^{-} X(t)+\frac{1}{2} \frac{\partial^{2} f}{\partial x^{2}}(t, X(t)) v^{2}(t) d t .
$$


We also need the following forward integral result, which is obtained by an adaptation of the proof of Theorem 8.18 in $[\mathrm{D} \varnothing \mathrm{P}]$ :

Proposition 2.8 Let $\varphi$ be a càglàd and forward integrable process in $L^{2}(\lambda \times P)$. Then

$$
\mathbb{E}\left[D_{s^{+}} \varphi(s) \mid \mathcal{F}_{s}\right]:=\lim _{\epsilon \rightarrow 0^{+}} \frac{1}{\epsilon} \int_{s-\epsilon}^{s} \mathbb{E}\left[D_{s} \varphi(t) \mid \mathcal{F}_{s}\right] d t
$$

exists in $L^{2}(\lambda \times P)$ and

$$
\mathbb{E}\left[\int_{0}^{T} \varphi(s) d^{-} B(s)\right]=\mathbb{E}\left[\int_{0}^{T} \mathbb{E}\left[D_{s^{+}} \varphi(s) \mid \mathcal{F}_{s}\right] d s\right] .
$$

where $D_{s} \varphi$ is the Hida-Malliavin derivative of $\varphi$ with respect to the Brownian motion B.

Similar definitions and results can be obtained in the Poisson random measure case. See $[\mathrm{DM} \varnothing \mathrm{P} 1]$ and $[\mathrm{D} \varnothing \mathrm{P}]$.

\subsection{The Donsker delta functional}

In this section we will define the Donsker delta function of a random variable $Y=\left(Y^{1}, Y^{2}\right)$ with value in $\mathbb{R}^{2}$, because in section 3 we want to prove that under the filtration $\mathbb{H}$ the knowledge of the value of $B\left(t+\varepsilon_{t}\right)$ is required to study the viability of the market. That is mean we will prove that if we know the values of $B\left(t+\varepsilon_{t}^{(1)}\right)$ and $B\left(t+\varepsilon_{t}^{(2)}\right)$ for $\varepsilon_{t}^{(1)}<\varepsilon_{t}^{(2)}$ then the maximal expected utility of the terminal wealth depends only on the knowledge of $B\left(t+\varepsilon_{t}^{(1)}\right)$.

Definition 2.9 Let $Y=\left(Y^{1}, Y^{2}\right): \Omega \rightarrow \mathbb{R}^{2}$ be a random variable which also belongs to the Hida space $(\mathcal{S})^{*}$ of stochastic distributions. Then a continuous functional

$$
\delta_{Y}(.): \mathbb{R}^{2} \rightarrow(\mathcal{S})^{*}
$$

$\{$ donsker $\}$

is called a Donsker delta functional of $Y$ if it has the property that

$$
\int_{\mathbb{R}^{2}} g(y) \delta_{Y}(y) d y=g(Y) \quad \text { a.s. }
$$

\{donsker

for all (measurable) $g: \mathbb{R}^{2} \rightarrow \mathbb{R}$ such that the integral converges. Here, and in the following, $d y=d y_{1} d y_{2}$ denotes 2-dimensional Lebesgue measure.

Proposition 2.10 [AaØU] Suppose $Y: \Omega \rightarrow \mathbb{R}^{2}$ is a normally distributed random variable with mean $m=\mathbb{E}[Y]$ and covariance matrix $C=\left(c_{i j}\right)_{1 \leq i, j \leq 2}$. Suppose $C$ is invertible with inverse $A=\left(a_{i j}\right)_{1 \leq i, j \leq 2}$. Then $\delta_{Y}(y)$ is unique and is given by the expression

$$
\delta_{Y}(y)=(2 \pi)^{-1}|A| \exp ^{\diamond}\left\{-\frac{1}{2} \sum_{i, j=1}^{2} a_{i j}\left(y_{i}-Y_{i}\right) \diamond\left(y_{j}-Y_{j}\right)\right\}
$$

where $|A|$ is the determinant of $A$ 
Explicit formulas for the Donsker delta functional are known in many cases. For the Gaussian case, see Section 2.3. For details and more general cases, see e.g. [AaØU], [DiØ1],[DiØ2],[MØP] and [DØ1].

In the next subsection we choose the particular example $Y(t)=\left(B\left(t+\epsilon_{t}^{(1)}\right), B\left(t+\epsilon_{t}^{(2)}\right)\right)$ where $\epsilon_{t}^{(1)}<\epsilon_{t}^{(2)}$ because in Section 3 we aim to prove that the knowledge of $B\left(t+\epsilon_{t}^{(1)}\right)$ is required to study the viability of the market.

Here are some useful formulas used in the calculation of Example 2.1:

Let $F$ and $G \in(\mathcal{S})^{*}$, we have:

$$
\begin{gathered}
D_{t}(F \diamond G)=F \diamond D_{t} G+D_{t} F \diamond G, \\
D_{t}\left(F^{\diamond n}\right)=n F^{\diamond(n-1)} \diamond D_{t} F, \quad(n=1,2 \ldots), \\
D_{t} \exp ^{\diamond} F=\exp ^{\diamond} F \diamond D_{t} F, \\
\mathbb{E}\left[\exp ^{\diamond} F \mid \mathcal{F}_{t}\right]=\exp \diamond \mathbb{E}\left[F \mid \mathcal{F}_{t}\right], \\
D_{t}\left(\int_{\mathbb{R}} f(s) d B(s)\right)=f(t) .
\end{gathered}
$$

\subsection{Examples}

Example 2.1 Let $Y(t)=\left(B\left(t+\epsilon_{t}^{(1)}\right), B\left(t+\epsilon_{t}^{(2)}\right)\right)$ where $\epsilon_{t}^{(1)}<\epsilon_{t}^{(2)}$. The expectation of $Y$ is given by $\mathbb{E}[Y(t)]=(0,0)$ and the variance matrix is

$$
V=\left[\begin{array}{cc}
t+\epsilon_{t}^{(1)} & t+\epsilon_{t}^{(1)} \\
t+\epsilon_{t}^{(1)} & t+\epsilon_{t}^{(2)}
\end{array}\right] .
$$

Its inverse matrix is given by

$$
A=\left[\begin{array}{cc}
\frac{t+\epsilon_{t}^{(2)}}{\left(t+\epsilon_{t}^{(1)}\right)\left(\epsilon_{t}^{(2)}-\epsilon_{t}^{(1)}\right)} & \frac{-1}{\epsilon_{t}^{(2)}-\epsilon_{t}^{(1)}} \\
\frac{-1}{\epsilon_{t}^{(2)}-\epsilon_{t}^{(1)}} & \frac{1}{\epsilon_{t}^{(2)}-\epsilon_{t}^{(1)}}
\end{array}\right] .
$$

The determinant of $A$ is given by:

$$
\operatorname{det}(A)=\frac{1}{\left(t+\epsilon_{t}^{(1)}\right)\left(\epsilon_{t}^{(2)}-\epsilon_{t}^{(1)}\right)}>0 .
$$

Then the Donsker delta of $Y(t)=\left(B\left(t+\epsilon_{t}^{(1)}\right), B\left(t+\epsilon_{t}^{(2)}\right)\right)$ is given by:

$$
\begin{gathered}
\delta_{B\left(t+\epsilon_{t}^{(1)}\right), B\left(t+\epsilon_{t}^{(2)}\right)}=(2 \pi)^{-1} \sqrt{\operatorname{det}(A)} \exp ^{\diamond}\left(-\frac{t+\epsilon_{t}^{(2)}}{2\left(t+\epsilon_{t}^{(1)}\right)\left(\epsilon_{t}^{(2)}-\epsilon_{t}^{(1)}\right)}\left(y_{1}-B\left(t+\epsilon_{t}^{(1)}\right)\right)^{\diamond 2}\right. \\
\left.\left.+\frac{1}{\epsilon_{t}^{(2)}-\epsilon_{t}^{(1)}}\left(y_{1}-B\left(t+\epsilon_{t}^{(1)}\right)\right) \diamond\left(y_{2}-B\left(t+\epsilon_{t}^{(2)}\right)\right)-\frac{1}{2\left(\epsilon_{t}^{(2)}-\epsilon_{t}^{(1)}\right)}\left(y_{2}-B\left(t+\epsilon_{t}^{(2)}\right)\right)^{\diamond 2}\right]\right) .
\end{gathered}
$$


Using the Wick rule when taking conditional expectation and using the martingale property of the process $B\left(t+\epsilon_{t}^{(1)}\right)$ and $B\left(t+\epsilon_{t}^{(2)}\right)$ we get

$$
\begin{aligned}
& \mathbb{E}\left[\delta_{Y}(y) \mid \mathcal{F}_{t}\right]=(2 \pi)^{-1} \sqrt{\operatorname{det}(A)} \exp ^{\diamond}\left(\mathbb{E}\left[-\frac{t+\epsilon_{t}^{(2)}}{2\left(t+\epsilon_{t}^{(1)}\right)\left(\epsilon_{t}^{(2)}-\epsilon_{t}^{(1)}\right)}\left(y_{1}-B\left(t+\epsilon_{t}^{(1)}\right)\right)^{\diamond 2} \mid \mathcal{F}_{t}\right]\right. \\
& +\mathbb{E}\left[\frac{1}{\epsilon_{t}^{(2)}-\epsilon_{t}^{(1)}}\left(y_{1}-B\left(t+\epsilon_{t}^{(1)}\right)\right) \diamond\left(y_{2}-B\left(t+\epsilon_{t}^{(2)}\right)\right) \mid \mathcal{F}_{t}\right] \\
& \left.-\mathbb{E}\left[\frac{1}{2\left(\epsilon_{t}^{(2)}-\epsilon_{t}^{(1)}\right)}\left(y_{2}-B\left(t+\epsilon_{t}^{(2)}\right)\right)^{\diamond 2} \mid \mathcal{F}_{t}\right]\right) \\
& =(2 \pi)^{-1} \sqrt{\operatorname{det}(A)} \exp ^{\diamond}\left(-\frac{t+\epsilon_{t}^{(2)}}{2\left(t+\epsilon_{t}^{(1)}\right)\left(\epsilon_{t}^{(2)}-\epsilon_{t}^{(1)}\right)}\left(y_{1}-B(t)\right)^{\diamond 2}\right. \\
& \left.+\frac{1}{\epsilon_{t}^{(2)}-\epsilon_{t}^{(1)}}\left(y_{1}-B(t)\right) \diamond\left(y_{2}-B(t)\right)-\frac{1}{2\left(\epsilon_{t}^{(2)}-\epsilon_{t}^{(1)}\right)}\left(y_{2}-B(t)\right)^{\diamond 2}\right) \\
& =(2 \pi)^{-1} \sqrt{\operatorname{det}(A)} \exp \left(-\frac{\left(y_{1}-y_{2}\right)^{2}}{2\left(\epsilon_{t}^{(2)}-\epsilon_{t}^{(1)}\right)}\right) \exp ^{\diamond}\left(-\frac{1}{2\left(t+\epsilon_{t}^{(1)}\right)}\left(y_{1}-B(t)\right)^{\diamond 2}\right) .
\end{aligned}
$$

To be with same notation as in [AaØU], we denote by $a=-\frac{1}{2\left(t+\epsilon_{t}^{(1)}\right)}$ and $\psi=1_{[0, t]}$.

We have $2|a|\|\psi\|=\frac{t}{t+\epsilon_{t}^{(1)}}<1$ then using Corollary 3.6 in [AaØU] we get

$$
\begin{aligned}
& \mathbb{E}\left[\delta_{Y}(y) \mid \mathcal{F}_{t}\right]=(2 \pi)^{-1} \exp \left(-\frac{\left(y_{1}-y_{2}\right)^{2}}{2\left(\epsilon_{t}^{(2)}-\epsilon_{t}^{(1)}\right)}\right) \sqrt{\frac{1}{\epsilon_{t}^{(1)}\left(\epsilon_{t}^{(2)}-\epsilon_{t}^{(1)}\right)}} \\
& \exp \left(-\frac{1}{2 \epsilon_{t}^{(1)}}\left(y_{1}-B(t)\right)^{2}\right) .
\end{aligned}
$$

Now we want to compute $\mathbb{E}\left[D_{t} \delta_{Y}(y) \mid \mathcal{F}_{t}\right]$. We have

$$
\begin{aligned}
& D_{t} \delta_{Y}(y)=D_{t}\left[( 2 \pi ) ^ { - 1 } \sqrt { \operatorname { d e t } ( A ) } \operatorname { e x p } ^ { \diamond } \left(-\frac{t+\epsilon_{t}^{(2)}}{2\left(t+\epsilon_{t}^{(1)}\right)\left(\epsilon_{t}^{(2)}-\epsilon_{t}^{(1)}\right)}\left(y_{1}-B\left(t+\epsilon_{t}^{(1)}\right)\right)^{\diamond 2}\right.\right. \\
& \left.\left.\left.+\frac{1}{\epsilon_{t}^{(2)}-\epsilon_{t}^{(1)}}\left(y_{1}-B\left(t+\epsilon_{t}^{(1)}\right)\right) \diamond\left(y_{2}-B\left(t+\epsilon_{t}^{(2)}\right)\right)-\frac{1}{2\left(\epsilon_{t}^{(2)}-\epsilon_{t}^{(1)}\right)}\left(y_{2}-B\left(t+\epsilon_{t}^{(2)}\right)\right)^{\diamond 2}\right]\right)\right] \\
& =(2 \pi)^{-1} \sqrt{\operatorname{det}(A)} \exp ^{\diamond}\left(-\frac{t+\epsilon_{t}^{(2)}}{2\left(t+\epsilon_{t}^{(1)}\right)\left(\epsilon_{t}^{(2)}-\epsilon_{t}^{(1)}\right)}\left(y_{1}-B\left(t+\epsilon_{t}^{(1)}\right)\right)^{\diamond 2}\right. \\
& \left.\left.+\frac{1}{\epsilon_{t}^{(2)}-\epsilon_{t}^{(1)}}\left(y_{1}-B\left(t+\epsilon_{t}^{(1)}\right)\right) \diamond\left(y_{2}-B\left(t+\epsilon_{t}^{(2)}\right)\right)-\frac{1}{2\left(\epsilon_{t}^{(2)}-\epsilon_{t}^{(1)}\right)}\left(y_{2}-B\left(t+\epsilon_{t}^{(2)}\right)\right)^{\diamond 2}\right]\right) \\
& \diamond\left\{\frac{t+\epsilon_{t}^{(2)}}{\left(t+\epsilon_{t}^{(1)}\right)\left(\epsilon_{t}^{(2)}-\epsilon_{t}^{(1)}\right)}\left(y_{1}-B\left(t+\epsilon_{t}^{(1)}\right)\right)\right. \\
& \left.-\frac{1}{\epsilon_{t}^{(2)}-\epsilon_{t}^{(1)}}\left(y_{1}-B\left(t+\epsilon_{t}^{(1)}\right)+y_{2}-B\left(t+\epsilon_{t}^{(2)}\right)\right)+\frac{1}{\epsilon_{t}^{(2)}-\epsilon_{t}^{(1)}}\left(y_{2}-B\left(t+\epsilon_{t}^{(2)}\right)\right)\right\}
\end{aligned}
$$


Then

$$
\begin{aligned}
& \mathbb{E}\left[D_{t} \delta_{Y}(y) \mid \mathcal{F}_{t}\right]=(2 \pi)^{-1} \sqrt{\operatorname{det}(A)} \mathbb{E}\left[\operatorname { e x p } ^ { \diamond } \left(-\frac{t+\epsilon_{t}^{(2)}}{2\left(t+\epsilon_{t}^{(1)}\right)\left(\epsilon_{t}^{(2)}-\epsilon_{t}^{(1)}\right)}\left(y_{1}-B\left(t+\epsilon_{t}^{(1)}\right)\right)^{\diamond 2}\right.\right. \\
& \left.\left.\left.+\frac{1}{\epsilon_{t}^{(2)}-\epsilon_{t}^{(1)}}\left(y_{1}-B\left(t+\epsilon_{t}^{(1)}\right)\right) \diamond\left(y_{2}-B\left(t+\epsilon_{t}^{(2)}\right)\right)-\frac{1}{2\left(\epsilon_{t}^{(2)}-\epsilon_{t}^{(1)}\right)}\left(y_{2}-B\left(t+\epsilon_{t}^{(2)}\right)\right)^{\diamond 2}\right]\right) \mid \mathcal{F}_{t}\right] \\
& \diamond \mathbb{E}\left[\frac{t+\epsilon_{t}^{(2)}}{\left(t+\epsilon_{t}^{(1)}\right)\left(\epsilon_{t}^{(2)}-\epsilon_{t}^{(1)}\right)}\left(y_{1}-B\left(t+\epsilon_{t}^{(1)}\right)\right)\right. \\
& \left.-\frac{1}{\epsilon_{t}^{(2)}-\epsilon_{t}^{(1)}}\left(y_{1}-B\left(t+\epsilon_{t}^{(1)}\right)+y_{2}-B\left(t+\epsilon_{t}^{(2)}\right)\right)+\frac{1}{\epsilon_{t}^{(2)}-\epsilon_{t}^{(1)}}\left(y_{2}-B\left(t+\epsilon_{t}^{(2)}\right)\right) \mid \mathcal{F}_{t}\right] \\
& =(2 \pi)^{-1} \sqrt{\frac{1}{\left(t+\epsilon_{t}^{(1)}\right)\left(\epsilon_{t}^{(2)}-\epsilon_{t}^{(1)}\right)}} \exp \left(-\frac{\left(y_{1}-y_{2}\right)^{2}}{2\left(\epsilon_{t}^{(2)}-\epsilon_{t}^{(1)}\right)}\right) \exp ^{\diamond}\left(-\frac{-\left(\epsilon_{t}^{(2)}-\epsilon_{t}^{(1)}\right)}{2\left(\epsilon_{t}^{(2)}-\epsilon_{t}^{(1)}\right)\left(t+\epsilon_{t}^{(1)}\right)}\left(y_{1}-B(t)\right)^{\diamond 2}\right) \\
& \diamond\left\{\frac{y_{1}-B(t)}{t+\epsilon_{t}^{(1)}}\right\} .
\end{aligned}
$$

By Lemma 3.8 in [AaØU]

$$
\begin{aligned}
& \frac{1}{\sqrt{t+\epsilon_{t}^{(1)}}} \exp ^{\diamond}\left(-\frac{1}{2\left(t+\epsilon_{t}^{(1)}\right)}\left(y_{1}-B(t)\right)^{\diamond 2}\right) \diamond \frac{y_{1}-B(t)}{t+\epsilon_{t}^{(1)}} \\
& =\frac{1}{\sqrt{\epsilon_{t}^{(1)}}} \exp \left(-\frac{\left(y_{1}-B(t)\right)^{2}}{2 \epsilon_{t}^{(1)}}\right) \frac{y_{1}-B(t)}{\epsilon_{t}^{(1)}} .
\end{aligned}
$$

Then

$$
\begin{aligned}
& \mathbb{E}\left[D_{t} \delta_{Y}(y) \mid \mathcal{F}_{t}\right]=(2 \pi)^{-1} \exp \left(-\frac{\left(y_{1}-y_{2}\right)^{2}}{2\left(\epsilon_{t}^{(2)}-\epsilon_{t}^{(1)}\right)}\right) \sqrt{\frac{1}{\epsilon_{t}^{(1)}\left(\epsilon_{t}^{(2)}-\epsilon_{t}^{(1)}\right)}} \\
& \exp \left(-\frac{\left(y_{1}-B(t)\right)^{2}}{2 \epsilon_{t}^{(1)}}\right) \frac{y_{1}-B(t)}{\epsilon_{t}^{(1)}} .
\end{aligned}
$$

\section{The main results with $t+\varepsilon_{t} \geq T$ for all $t$}

In this section we prove a necessary condition for the viability of the market when $t+\varepsilon_{t} \geq T$ for all $t$. To this end, we first consider the case when the inside information is given by

$$
Y_{t}=\left(B\left(t+\varepsilon_{t}^{(1)}\right), B\left(t+\varepsilon_{t}^{(2)}\right)\right) ; \quad t \in(0, T)
$$

for $\varepsilon_{t}^{(1)}<\varepsilon_{t}^{(2)}$.

Then we have the following result, which is of independent interest: 
Theorem 3.1 The maximal expected logarithmic utility of Problem 1.1 in the insider market (1.2)-(1.4) with inside information $Y_{t}=\left(B\left(t+\epsilon_{t}^{1}\right), B\left(t+\epsilon_{t}^{2}\right)\right)$ is

$$
\mathbb{E}\left[\log X^{\pi^{*}}(T)\right]=\frac{1}{2} \mathbb{E}\left[\int_{0}^{T}\left(\frac{1}{\varepsilon_{t}^{(1)}}+\left(\frac{\alpha(t)}{\beta(t)}\right)^{2}\right) d t\right] .
$$

Proof.

This type of problem is studied in [DØ1] and [ØR]. In [DØ1] it is proved that the optimal insider portfolio $\pi^{*}\left(t, Y_{t}\right)$ with $Y_{t}=\left(B\left(t+\varepsilon_{t}^{(1)}\right), B\left(t+\varepsilon_{t}^{(2)}\right)\right)$ of Problem 1.1 is given by

$$
\pi^{*}\left(t, Y_{t}\right)=\frac{\alpha(t)}{\beta^{2}(t)}+\frac{\mathbb{E}\left[D_{t^{+}} \delta_{Y_{t}}(y) \mid \mathcal{F}_{t}\right]_{y=Y_{t}}}{\beta(t) \mathbb{E}\left[\delta_{Y_{t}}(y) \mid \mathcal{F}_{t}\right]_{y=Y_{t}}}
$$

$\{$ eq3.3ench $\}$

In terms of $y$ we have:

$$
\pi^{*}(t, y)=\frac{\alpha(t)}{\beta^{2}(t)}+\frac{\mathbb{E}\left[D_{t^{+}} \delta_{Y_{t}}(y) \mid \mathcal{F}_{t}\right]}{\beta(t) \mathbb{E}\left[\delta_{Y_{t}}(y) \mid \mathcal{F}_{t}\right]} .
$$

We have, with $y=\left(y_{1}, y_{2}\right)$,

$$
\begin{aligned}
& \mathbb{E}\left[\delta_{Y}(y) \mid \mathcal{F}_{t}\right] \\
& =(2 \pi)^{-1} \exp \left(-\frac{\left(y_{1}-y_{2}\right)^{2}}{2\left(\varepsilon_{t}^{(2)}-\varepsilon_{t}^{(1)}\right)}\right) \sqrt{\frac{1}{\varepsilon_{t}^{(1)}\left(\varepsilon_{t}^{(2)}-\varepsilon_{t}^{(1)}\right)}} \exp \left(-\frac{1}{2 \varepsilon_{t}^{(1)}}\left(y_{1}-B(t)\right)^{2}\right),
\end{aligned}
$$

and

$$
\begin{aligned}
& \mathbb{E}\left[D_{t} \delta_{Y}(y) \mid \mathcal{F}_{t}\right] \\
& =(2 \pi)^{-1} \exp \left(-\frac{\left(y_{1}-y_{2}\right)^{2}}{2\left(\varepsilon_{t}^{(2)}-\varepsilon_{t}^{(1)}\right)}\right) \sqrt{\frac{1}{\varepsilon_{t}^{(1)}\left(\varepsilon_{t}^{(2)}-\varepsilon_{t}^{(1)}\right)}} \exp \left(-\frac{\left(y_{1}-B(t)\right)^{2}}{2 \varepsilon_{t}^{(1)}}\right) \frac{y_{1}-B(t)}{\varepsilon_{t}^{(1)}} .
\end{aligned}
$$

Substituting (3.5) and (3.6) in (3.4), we get

$$
\pi^{*}(t, y)=\frac{\alpha(t)}{\beta^{2}(t)}-\frac{B(t)-y_{1}}{\beta(t) \varepsilon_{t}^{(1)}} .
$$

The solution of the stochastic differential equation (1.2) is given by:

$$
X(t)=\exp \left[\int_{0}^{t} \pi\left(s, Y_{s}\right) \beta(s) d B(s)+\int_{0}^{t}\left(\pi\left(s, Y_{s}\right) \alpha(s)-\frac{1}{2} \pi^{2}\left(s, Y_{s}\right) \beta^{2}(s)\right) d s\right] .
$$

Using the Donsker delta functional, Hida-Malliavin derivative and the following duality formula for forward integrals:

$$
\mathbb{E}\left[\int_{0}^{T} \phi(t) d B(t)\right]=\mathbb{E}\left[\int_{0}^{T} \mathbb{E}\left[D_{t^{+}} \phi(t) \mid \mathcal{F}_{t}\right] d t\right],
$$


we get

$$
\begin{aligned}
& \mathbb{E}\left[\log X^{\pi}(T)\right]=\int_{\mathbb{R}^{2}} \mathbb{E}\left[\int _ { 0 } ^ { T } \left\{\pi(t, y) \beta(t) \mathbb{E}\left[D_{t^{+}} \delta_{Y_{t}}(y) \mid \mathcal{F}_{t}\right]+\pi(t, y) \alpha(t) \mathbb{E}\left[\delta_{Y_{t}}(y) \mid \mathcal{F}_{t}\right]\right.\right. \\
& \left.\left.-\frac{1}{2} \pi^{2}(t, y) \beta^{2}(t) \mathbb{E}\left[\delta_{Y_{t}}(y) \mid \mathcal{F}_{t}\right]\right\} d t\right] d y .
\end{aligned}
$$

For more details about equation (3.10) see [D $\varnothing 1]$.

Substituting (3.7) in (3.10) we get

$$
\begin{aligned}
& \int_{\mathbb{R}^{2}} \mathbb{E}\left[\int _ { 0 } ^ { T } \left\{\left[\frac{\alpha(t)}{\beta^{2}(t)}-\frac{B(t)-y_{1}}{\beta(t) \varepsilon_{t}^{(1)}}\right] \beta(t) \mathbb{E}\left[D_{t^{+}} \delta_{Y_{t}}(y) \mid \mathcal{F}_{t}\right]+\left[\frac{\alpha(t)}{\beta^{2}(t)}-\frac{B(t)-y_{1}}{\beta(t) \varepsilon_{t}^{(1)}}\right] \alpha(t) \mathbb{E}\left[\delta_{Y_{t}}(y) \mid \mathcal{F}_{t}\right]\right.\right. \\
& \left.\left.-\frac{1}{2}\left[\frac{\alpha(t)}{\beta^{2}(t)}-\frac{B(t)-y_{1}}{\beta(t) \varepsilon_{t}^{(1)}}\right]^{2} \beta^{2}(t) \mathbb{E}\left[\delta_{Y_{t}}(y) \mid \mathcal{F}_{t}\right]\right\} d t\right] d y \\
& =\mathbb{E}\left[\int _ { 0 } ^ { T } \left\{\int_{\mathbb{R}}\left(\int_{\mathbb{R}}(2 \pi)^{-\frac{1}{2}} \sqrt{\frac{1}{\left(\epsilon_{t}^{(2)}-\epsilon_{t}^{(1)}\right)}} \exp \left(-\frac{\left(y_{1}-y_{2}\right)^{2}}{2\left(\epsilon_{t}^{(2)}-\epsilon_{t}^{(1)}\right)}\right) d y_{2}\right)\right.\right. \\
& \times\left(2 \pi \epsilon_{t}^{(1)}\right)^{-\frac{1}{2}} \exp \left(-\frac{\left(y_{1}-B(t)\right)^{2}}{2 \epsilon_{t}^{(1)}}\right)\left[\frac{\alpha(t)}{\beta^{2}(t)}-\frac{B(t)-y_{1}}{\beta(t) \varepsilon_{t}^{(1)}}\right] \\
& \left.\left.\left(\beta(t) \frac{y_{1}-B(t)}{\epsilon_{t}^{(1)}}+\alpha(t)-\frac{1}{2} \beta^{2}(t)\left[\frac{\alpha(t)}{\beta^{2}(t)}-\frac{B(t)-y_{1}}{\beta(t) \varepsilon_{t}^{(1)}}\right]\right) d y_{1}\right\}\right] \\
& =\frac{1}{2}\left(2 \pi \varepsilon_{t}^{(1)}\right)^{-\frac{1}{2}} \int_{\mathbb{R}} \mathbb{E}\left[\int_{0}^{T} \exp \left(-\frac{\left(B(t)-y_{1}\right)^{2}}{2 \varepsilon_{t}^{(1)}}\right)\left(\frac{B(t)-y_{1}}{\varepsilon_{t}^{(1)}}-\frac{\alpha(t)}{\beta(t)}\right)^{2} d t\right] d y_{1} .
\end{aligned}
$$

Put

$$
b=B(t) \text { and } \sigma=\frac{\alpha(t)}{\beta(t)} \text {. }
$$


Integrating the above with respect to $d y_{1}$ we get:

$$
\begin{aligned}
& \left(2 \pi \varepsilon_{t}\right)^{-\frac{1}{2}} \int_{\mathbb{R}} \exp \left(-\frac{\left(B(t)-y_{1}\right)^{2}}{2 \varepsilon_{t}^{(1)}}\right)\left(\frac{B(t)-y_{1}}{\varepsilon_{t}^{(1)}}-\frac{\alpha(t)}{\beta(t)}\right)^{2} d y_{1} \\
& =\left(2 \pi \varepsilon_{t}^{(1)}\right)^{-\frac{1}{2}} \int_{\mathbb{R}} \exp \left(-\frac{\left(b-y_{1}\right)^{2}}{2 \varepsilon_{t}^{(1)}}\right)\left(\frac{b-y_{1}}{\varepsilon_{t}^{(1)}}-\sigma\right)^{2} d y_{1} \\
& =\left(2 \pi \varepsilon_{t}^{(1)}\right)^{-\frac{1}{2}} \int_{\mathbb{R}} \exp \left(-\frac{\left(b-y_{1}\right)^{2}}{2 \varepsilon_{t}^{(1)}}\right)\left(\frac{b-y_{1}}{\varepsilon_{t}^{(1)}}-\sigma\right)\left(\frac{b-y_{1}}{\varepsilon_{t}^{(1)}}-\sigma\right) d y_{1} \\
& =\left(2 \pi \varepsilon_{t}^{(1)}\right)^{-\frac{1}{2}} \int_{\mathbb{R}}\left\{\frac{d}{d y_{1}} \exp \left(-\frac{\left(b-y_{1}\right)^{2}}{2 \varepsilon_{t}^{(1)}}\right)-\sigma \exp \left(-\frac{\left(b-y_{1}\right)^{2}}{2 \varepsilon_{t}^{(1)}}\right)\right\}\left(\frac{b-y_{1}}{\varepsilon_{t}^{(1)}}-\sigma\right) d y_{1} \\
& =\left(2 \pi \varepsilon_{t}^{(1)}\right)^{-\frac{1}{2}}\left[\int_{\mathbb{R}} \frac{d}{d y_{1}} \exp \left(-\frac{\left(b-y_{1}\right)^{2}}{2 \varepsilon_{t}^{(1)}}\right)\left(\frac{b-y_{1}}{\varepsilon_{t}^{(1)}}-\sigma\right) d y_{1}+\sigma^{2} \int_{\mathbb{R}} \exp \left(-\frac{\left(b-y_{1}\right)^{2}}{2 \varepsilon_{t}^{(1)}}\right) d y_{1}\right] \\
& =\left(2 \pi \varepsilon_{t}^{(1)}\right)^{-\frac{1}{2}}\left[\int_{\mathbb{R}} \exp \left(-\frac{\left(b-y_{1}\right)^{2}}{2 \varepsilon_{t}^{(1)}}\right)\left(\frac{1}{\varepsilon_{t}^{(1)}}\right) d y_{1}+\sigma^{2} \int_{\mathbb{R}} \exp \left(-\frac{\left(b-y_{1}\right)^{2}}{2 \varepsilon_{t}^{(1)}}\right) d y_{1}\right] \\
& =\frac{1}{\varepsilon_{t}^{(1)}}+\sigma^{2}=\frac{1}{\varepsilon_{t}^{(1)}}+\left(\frac{\alpha(t)}{\beta(t)}\right)^{2} .
\end{aligned}
$$

Then from equation (3.11) we get:

$$
\mathbb{E}\left[\log X^{\pi^{*}}(T)\right]=\frac{1}{2} \mathbb{E}\left[\int_{0}^{T}\left(\frac{1}{\varepsilon_{t}^{(1)}}+\left(\frac{\alpha(t)}{\beta(t)}\right)^{2}\right) d t\right],
$$

which is what we wanted to prove.

Remark 3.2 From this Theorem we get that the maximal expected utility of the terminal wealth depends only on the value of $B\left(t+\varepsilon_{t}^{(1)}\right)$. Thus the additional information about the value of $B\left(t+\varepsilon_{t}^{(2)}\right)$ is irrelevant for the optimisation problem if we already know $B\left(t+\varepsilon_{t}^{(1)}\right)$. This is a strong indication that if the insider already knows the value of $B\left(t+\varepsilon_{t}\right)$, then knowing in addition the values of $B(t+r)$ for all $\left.r \in] \varepsilon_{t}, \varepsilon_{0}-t\right]$ does not increase the value of the optimal portfolio. However, we have not been able to prove this.

As a corollary of Theorem 3.1, we get the following necessary condition for viability:

Theorem 3.3 This insider market (0.1), (1.2)-(1.4) is viable only if

$$
\int_{0}^{T} \frac{1}{\varepsilon_{t}} d t<\infty
$$

Remark 3.4 As noted in Remark 3.2, Theorem 3.1 supports our conjecture that we in fact have that the market is viable if and only if (3.15) holds. 
Example 3.1 1. If $\varepsilon_{t}=(T-t)^{q}$ for some $q>1$, then

$$
\int_{0}^{T} \frac{1}{\varepsilon_{t}} d t=\int_{0}^{T} \frac{1}{(T-t)^{q}} d t=\infty .
$$

and the market is not viable.

In particular, for $q=1$ (corresponding to $t+\varepsilon_{t}=T$ for all $t$ ), this was first proved by $[P K]$ by different methods. In this case $B(\cdot)$ is a semimartingale with respect to $\mathbb{H}:=\mathbb{K}:=\{\mathcal{K}\}_{t \geq 0}$, where $\mathcal{K}_{t}=\mathcal{F}_{t} \vee \sigma(B(T)), \forall t \in[0, T]$.

2. If $\varepsilon_{t}=(T-t)^{p}$ for some $p<1$, then

$$
\int_{0}^{T} \frac{1}{\varepsilon_{t}} d t=\int_{0}^{T}(T-t)^{-p} d t=\frac{T^{1-p}}{1-p}<\infty .
$$

In view of Theorem 3.3 this indicates that the market is viable.

\section{The case when $t+\varepsilon_{t} \leq T$ for all $t$}

If $t+\varepsilon_{t} \leq T$ for all $t$, then the natural corresponding information filtration for an insider with perfect memory is

$$
\mathbb{H}=\left\{\mathcal{H}_{t}\right\}_{t \geq 0} \text { where } \mathcal{H}_{t}=\mathcal{F}_{t+\varepsilon_{t}} .
$$

In this case $B(\cdot)$ is not a semimartingale with respect to $\mathbb{H}$ (see below). Nevertheless, our calculation above shows the following:

Theorem 4.1 The insider market (1.2)-(1.4), (4.1) is viable if and only if

$$
\int_{0}^{T} \frac{1}{\varepsilon_{t}} d t<\infty
$$

Since in this case $\varepsilon_{t} \leq T-t$ and $\int_{0}^{T} \frac{d t}{T-t}=\infty$, we conclude that the market is never viable in this case. This is also a generalisation of the [PK] result, but in a different direction.

Proposition 4.2 Suppose that $t \rightarrow \varepsilon_{t}$ is of finite variation. Then $B(\cdot)$ is not a semimartingale with respect to $\mathbb{H}$ where $\mathcal{H}_{t}=\mathcal{F}_{t+\varepsilon_{t}}, \quad \forall t \geq 0$.

Proof. $\quad$ Suppose that $B(\cdot)$ is an $\mathbb{H}$-semimartingale. Then

$$
B(t)=\tilde{B}(t)+A(t),
$$

where $\tilde{B}(t)$ is an $\mathbb{H}$ - martingale and $A(t)$ is an $\mathbb{H}$-adapted finite variation process. Then, for $t+\varepsilon_{t} \leq t+h \leq T$,

$$
\begin{aligned}
0 & =\mathbb{E}\left[\tilde{B}(t+h)-\tilde{B}(t) \mid \mathcal{H}_{t}\right] \\
& =\mathbb{E}\left[B(t+h)-B(t) \mid \mathcal{H}_{t}\right]-\mathbb{E}\left[A(t+h)-A(t) \mid \mathcal{H}_{t}\right] \\
& =\mathbb{E}\left[B(t+h)-B(t) \mid \mathcal{F}_{t+\varepsilon_{t}}\right]-\mathbb{E}\left[A(t+h)-A(t) \mid \mathcal{H}_{t}\right] \\
& =B\left(t+\varepsilon_{t}\right)-B(t)-\mathbb{E}\left[A(t+h)-A(t) \mid \mathcal{H}_{t}\right] .
\end{aligned}
$$


Then we get

$$
B\left(t+\varepsilon_{t}\right)-B(t)=\mathbb{E}\left[A(t+h)-A(t) \mid \mathcal{H}_{t}\right] .
$$

This is a contradiction, because $A$ is a finite variation process.

\section{References}

[AaØPU] K. Aase, B. Øksendal, N. Privault and J. Ubøe: White noise generalizations of the Clark-Haussmann-Ocone theorem with application to mathematical finance. Finance Stoch. 4 (2000), 465-496.

[AaØU] K. Aase, B. Øksendal and J. Ubøe: Using the Donsker delta function to compute hedging strategies. Potential Analysis 14 (2001), 351-374.

[A] J. Amendinger: Initial enlargement of filtrations and additional information in financial markets. Technischen Universität Berlin, (PhD Thesis) 1999.

[AIS] J. Amendinger, P. Imkeller, and M. Schweizer: Additional logarithmic utility of an insider. Stochastic Processes and their Appl, 75 (1998), 263-286.

[AI] S. Ankirchner, and P. Imkeller: Financial markets with asymmetric information: information drift, additional utility and entropy. Proceedings of the 6th Ritsumeikan International Symposium, 2007, 1-21.

[B] L. Breiman: Probability. Addison-Wesley 1968.

[BØ] F. Biagini and B. Øksendal: A general stochastic calculus approach to insider trading. Appl. Math. \& Optim. 52 (2005), 167-181.

[C] J.M. Corcuera, P. Imkeller, A. Kohatsu-Higa, and D. Nualart: Additional utility of insiders with imperfect dynamical information. Finance and Stochastics, 8 (2004), 437-450.

[DMØP1] G. Di Nunno, T. Meyer-Brandis, B. Øksendal and F. Proske: Malliavin calculus and anticipative Itô formulae for Lévy processes. Inf. Dim. Anal. Quantum Prob. Rel. Topics 8 (2005), 235-258.

[DMØP2] G. Di Nunno, T. Meyer-Brandis, B. Øksendal and F. Proske: Optimal portfolio for an insider in a market driven by Lévy processes. Quant. Finance 6 (2006), 83-94.

[DØPP] G. Di Nunno, B. Øksendal, O. M. Pamen and F. Proske: A general maximum principle for anticipative stochastic control and applications to insider trading. In $\mathrm{G}$. Di Nunno and B. Øksendal (editors): Advanced Mathematical Methods for Finance. Springer 2011, pp. 181-221. 
[DØ1] O. Draouil and B. Øksendal: A Donsker delta functional approach to optimal insider control and application to finance. Comm. Math. Stat. (CIMS) 3 (2015), 365-421; DOI 10.1007/s40304-015-0065-y.

[DØ2] O. Draouil and B. Øksendal: Optimal insider control and semimartingale decompositions under enlargement of filtration. arXiv: 1512.01759v1 (6 Dec.2015). To appear in Stochastic Analysis and Applications.

[DiØ1] G. Di Nunno and B. Øksendal: The Donsker delta function, a representation formula for functionals of a Lévy process and application to hedging in incomplete markets. Séminaires et Congrèes, Societé Mathématique de France, Vol. 16 (2007), 71-82.

[DiØ2] G. Di Nunno and B. Øksendal: A representation theorem and a sensitivity result for functionals of jump diffusions. In A.B. Cruzeiro, H. Ouerdiane and N. Obata (editors): Mathematical Analysis and Random Phenomena. World Scientific 2007, pp. $177-190$.

[DØP] G. Di Nunno, B. Øksendal and F. Proske: Malliavin Calculus for Lévy Processes with Applications to Finance. Universitext, Springer 2009.

$[\mathrm{H}]$ C. Hillairet: Existence of an equilibrium on a financial market with discontinuous prices, asymmetric information and non trivial initial $\sigma$-fields. Mathematical Finance, Vol. 15, No 1, January 2005, 99-117.

[Ja] J. Jacod: Grossissement initial, hypothese (H') et théorème de Girsanov , in Grossissements de filtrations: exemples et applications, Springer, 1985, pp. 15-35.

[J] M. Jeanblanc: Enlargements of filtrations. Lecture Notes, Jena 2010.

[Je] T. Jeulin, Semi-Martingales et Grossissement d'une Filtration, vol. 833 of Lecture Notes in Mathematics, Springer, 1980.

[IPW] P. Imkeller, M. Pontier and F. Weisz, Free lunch and arbitrage possibilities in a financial market model with an insider, Stochastic Processes and their Applications, 92 (2001), pp. 103-130.

[MØP] S. Mataramvura, B. Øksendal and F. Proske: The Donsker delta function of a Lévy process with application to chaos expansion of local time. Ann. Inst H. Poincaré Prob. Statist. 40 (2004), 553-567.

[M] P-A. Meyer: Sur un theoreme de J. Jacod, Séminaire de probabilités de Strasbourg, 12 (1978), pp. 57-60.

[ØPZ] B. Øksendal, F. Proske and T. Zhang: Backward stochastic partial differential equations with jumps and application to optimal control of random jump fields. Stochastics 77 (2005), 381-399. 
[ØR] B. Øksendal and E.Røse: A white noise approach to insider trading. In T.Hida and L.Streit(editors): Let Us Use White Noise. World Scientific 2017, ISBN 978-981-322093-5, pp.191-203.

[ØS1] B. Øksendal and A. Sulem: Applied Stochastic Control of Jump Diffusions. Second Edition. Springer 2007

[ØS2] B. Øksendal and A. Sulem: Risk minimization in financial markets modeled by ItôLévy processes. Afrika Matematika (2014), DOI: 10.1007/s13370-014-02489-9.

[P] P. Protter: Stochastic Integration and Differential Equations. Second Edition. Springer 2005

[PK] I. Pikovsky and I. Karatzas: Anticipative portfolio optimization. Adv. Appl. Probab. 28 (1996), 1095-1122.

[RV] F. Russo and P. Vallois: Forward, backward and symmetric stochastic integration. Probab. Theor. Rel. Fields 93 (1993), 403-421.

[RV1] F. Russo and P. Vallois. The generalized covariation process and Itô formula. Stoch. Proc. Appl., 59(4):81-104, 1995.

[RV2] F. Russo and P. Vallois. Stochastic calculus with respect to continuous finite quadratic variation processes. Stoch. Stoch. Rep., 70(4):1-40, 2000. 